\title{
Oxide Resistive Memory with Functionalized Graphene as Built-in Selector Element
}

\author{
Yuchao Yang, Jihang Lee, Seunghyun Lee, Che-Hung Liu, Zhaohui Zhong, and Wei Lu**
}

Driven by the continuing demand for improved computing capability, the semiconductor industry has been constantly looking for a fast, reliable, scalable yet nonvolatile memory technology. Nanoscale resistive switching devices (RRAMs) have recently generated significant interest as a potential building block for novel data storage and computing applications ${ }^{[1-7]}$ and have shown exciting performance metrics including endurance of $>10^{12},{ }^{[8]}$ programming time of $<1 \mathrm{~ns},{ }^{\left[{ }^{[9]}\right.}$ scalability better than $10 \mathrm{~nm}^{[10]}$ and retention of $>10$ years. ${ }^{[8]}$ In addition, since RRAM devices do not rely on conventional Si-based transistors, they can potentially be fabricated in three-dimensional (3D) multistack fashion or on diverse substrates for flexible and/or transparent electronics applications. ${ }^{[11,12]}$ This is particularly suitable for oxide-based valency-change type devices, where the resistance change is caused by the redistribution of oxygen vacancies inside the switching layer so potentially any inert metal can be used as the electrode material. ${ }^{[13]}$ To this end, instead of using conventional metal electrodes RRAM devices can be integrated with novel electrode materials such as graphene to take advantage of the excellent electrical, mechanical and optical properties of graphene. ${ }^{[14,15]}$ Furthermore, graphene can be readily functionalized or modified to exhibit a number of interesting electrical characteristics, ${ }^{[16-18]}$ and resistive switching effects in some graphene based materials, for example graphene oxide, have also been reported..$^{19-21]}$ In this study, we show that RRAM devices can be successfully integrated with graphene electrodes in a compact device structure. More importantly, functionalized graphene electrodes exhibit an intrinsic threshold switching behavior analogous to insulator-metal transition (IMT), and provide a built-in selector element for the RRAM devices that is very desirable for mitigating the sneak path problem in crossbar memory arrays. ${ }^{[22,23]}$

The devices studied in this work were arranged in a crossbar structure and fabricated on a glass substrate. Figure 1a schematically illustrates the fabrication process. Starting from a glass substrate, monolayer graphene films grown by chemical vapor deposition were transferred from the growth substrate (copper foil) onto glass by typical polymethyl methacrylate (PMMA) + etchant transfer method (Figure 1a1, Experimental Section). ${ }^{[24]}$ The sheet resistance of the graphene monolayer used in this study is $\sim 600 \Omega / \square$. The monolayer graphene

Dr. Y. Yang, J. Lee, Dr. S. Lee, C.-H. Liu, Prof. Z. Zhong,

Prof. W. Lu

Department of Electrical Engineering

and Computer Science

University of Michigan

Ann Arbor, Michigan 48109

E-mail:wluee@eecs.umich.edu

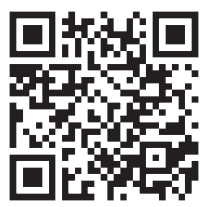

DOI: 10.1002/adma.201400270 transfer was repeated several times (typically $\sim 3$ ) in order to further improve the conductance of the electrodes, therefore forming a multilayer graphene (MLG) stack. An optical graph of the glass substrate with the MLG film on top is displayed in Figure 1b, showing high transparency. Subsequently, the MLG film was patterned into bottom electrodes (BEs) with widths of 1-5 $\mu \mathrm{m}$ by photolithography and $\mathrm{O}_{2}$ plasma etching (Figure 1a2). Au/Ti metal contacts (B1 and B2) were then deposited onto the two ends of the MLG BE afterwards to minimize the contact resistance to the external probes in electrical measurements (Figure 1a3). Following BE definition, a bilayer oxide structure consisting of an oxygen rich $\mathrm{Ta}_{2} \mathrm{O}_{5-x}$ layer $(\sim 5 \mathrm{~nm})$ and an oxygen deficient $\mathrm{TaO}_{\mathrm{y}}$ layer $(\sim 40 \mathrm{~nm})$ serving as the switching medium of the RRAM devices was successively deposited by radio-frequency (RF) sputtering and reactive sputtering $\left(400{ }^{\circ} \mathrm{C}, \mathrm{O}_{2} / \mathrm{Ar}=3 \%\right)$, respectively, without breaking the vacuum (Figure 1a4). Similar to steps 1-3 in Figure 1a, multiple monolayer graphene transfer, $\mathrm{O}_{2}$ plasma etching and $\mathrm{Au} / \mathrm{Ti}$ metal contacts deposition were conducted again to fabricate the top electrodes (TEs) that complete the crossbar memory structure (Figure 1a5-7). Finally, a pad opening step was performed through a timed reactive ion etching (RIE) process to remove the $\mathrm{Ta}_{2} \mathrm{O}_{5-x} / \mathrm{TaO}_{\mathrm{y}}$ bilayer on top of the bottom contact $\mathrm{Au} / \mathrm{Ti}$ pads (Figure 1a8). The sizes of the RRAM devices in this work range from 1-5 $\mathrm{mm} \times 1-5 \mu \mathrm{m}$, and during measurements the voltage was applied on the TE with the BE grounded. Figure $1 \mathrm{c}$ shows an optical graph of the as-fabricated chip. Compared with Figure 1b, the transparency decrease is likely due to the relatively high concentration of metallic components in the $\mathrm{TaO}_{\mathrm{y}}$ base layer of the switching medium.

Since resistive switching in $\mathrm{Ta}_{2} \mathrm{O}_{5-x} / \mathrm{TaO}_{y}$ bilayer devices is driven by internal oxygen vacancy redistribution, the change in deposition sequence should in principle only lead to a reversal of the switching polarity, as has been verified by studies on devices with inert metal electrodes. ${ }^{[25]}$ However, in the case of devices with MLG electrodes, the stacking sequence of the bilayer was found to be critical in determining the device behavior, as shown in Figure 1 d,e. When the $\mathrm{Ta}_{2} \mathrm{O}_{5-x}$ layer was deposited first on the graphene $\mathrm{BE}$ followed by $\mathrm{TaO}_{\mathrm{y}}$ deposition, the MLG/ $\mathrm{TaO}_{y} / \mathrm{Ta}_{2} \mathrm{O}_{5-x} / \mathrm{MLG}$ (top to bottom) devices exhibited conventional bipolar resistive switching characteristics similar to the results obtained with metal electrodes, ${ }^{[25,26]}$ as shown in Figure 1d. Such switching behavior can be well understood in the picture of $\mathrm{V}_{\mathrm{O}}$ exchange between the $\mathrm{Ta}_{2} \mathrm{O}_{5-x}$ and the $\mathrm{V}_{\mathrm{O}}$-rich $\mathrm{TaO}_{\mathrm{y}}$ layers, ${ }^{[8,26,27]}$ and the switching polarity with positive SET and negative RESET voltages is a natural result of the device configuration since the $\mathrm{V}_{\mathrm{O}}$ reservoir (the oxygen deficient $\mathrm{TaO}_{\mathrm{y}}$ layer) sits on top in this case. The linear on-state behavior is also consistent with Ohmic conduction for the $\mathrm{V}_{\mathrm{O}}$-based conducting filaments in $\mathrm{Ta}_{2} \mathrm{O}_{5-x} / \mathrm{TaO}_{\mathrm{y}}$ RRAMs. ${ }^{[8,26,27]}$ 
(a) 1

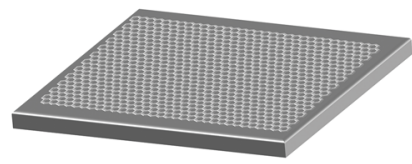

5

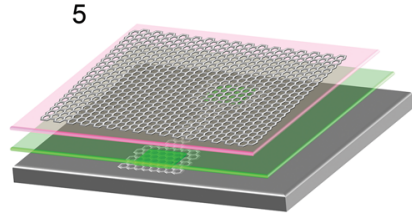

(b)

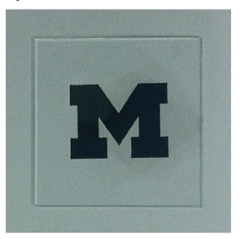

2

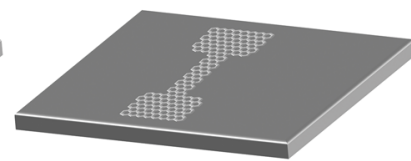

6

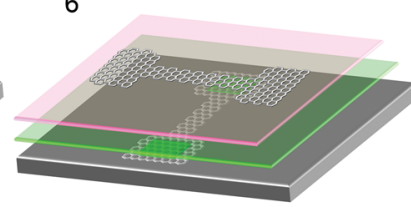

3

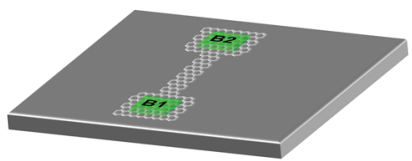

7

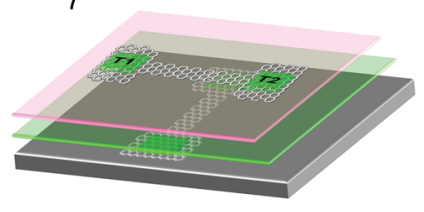

4
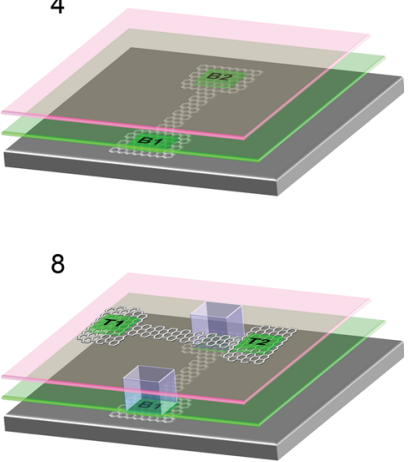

(c)

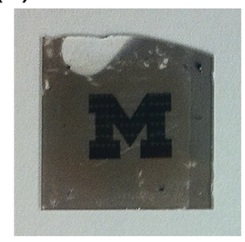

(d)

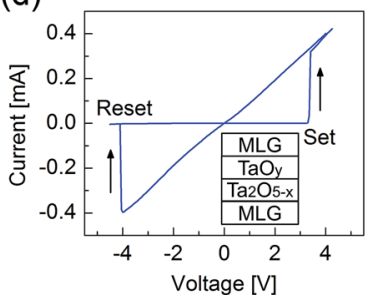

(e)

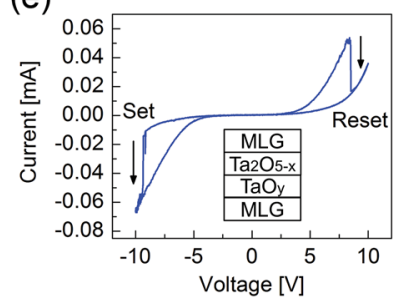

(f)

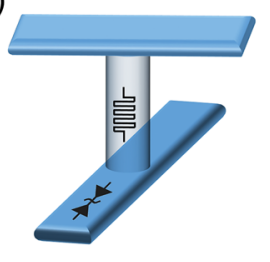

Figure 1. $\mathrm{Ta}_{2} \mathrm{O}_{5-x} / \mathrm{TaO}_{y}$ bilayer RRAM devices with graphene electrodes. (a) Schematic illustration of the fabrication process. (b) Optical graph of the glass substrate after MLG was transferred. (c) Optical graph of the glass chip after device fabrication. (d) Bipolar resistive switching characteristics of a MLG $/ \mathrm{TaO}_{y} / \mathrm{Ta}_{2} \mathrm{O}_{5-x} / \mathrm{MLG}$ (top to bottom) device, showing linear on-state characteristics. (e) Bipolar resistive switching characteristics of a MLG/ $\mathrm{Ta}_{2} \mathrm{O}_{5-x} / \mathrm{TaO}_{y} / \mathrm{MLG}$ device, showing significant on-state nonlinearity. (f) Schematic of the equivalent $1 \mathrm{~S} 1 \mathrm{R}$ structure formed in the $\mathrm{MLG} / \mathrm{Ta}_{2} \mathrm{O}_{5-x} / \mathrm{TaO}_{y} /$ MLG devices with an intrinsic selector element provided by the graphene electrode.

On the other hand, when the stacking sequence of the oxide bilayer was reversed, besides the expected reversal of the switching polarity, the MLG/ $\mathrm{Ta}_{2} \mathrm{O}_{5-x} / \mathrm{TaO}_{\mathrm{y}} / \mathrm{MLG}$ devices exhibited pronounced on-state nonlinearity, as shown in Figure 1e. A large non-linearity ratio $I\left(V_{\text {read }}\right) / I\left(V_{\text {read }} / 2\right)$ of $\sim 280$ can be obtained with a $V_{\text {read }}=-6.5 \mathrm{~V}$. Such an on-state non-linearity is highly desirable for suppressing the sneak path problem in passive crossbar arrays, ${ }^{[25,28]}$ and obtaining a non-linear RRAM cell with either intrinsic non-linear characteristics or with seriallyconnected selector devices have now become a major research direction. ${ }^{[4,29,30]}$ The integrated graphene/oxide RRAM structure with intrinsic non-linear switching characteristics thus offers an interesting option for not only flexible/transparent electronic applications but also high-performance RRAM applications in general. Similar results can be obtained in 54 devices from 3 batches, and the device yield was $\sim 60 \%$.

The non-linear on-state behavior cannot be simply explained by the reversal of the device layout, nor is the non-linear on-state behavior intrinsic to the $\mathrm{Ta}_{2} \mathrm{O}_{5-x} / \mathrm{TaO}_{y}$ bilayer switching stack. Studies on such devices with metal electrodes have consistently reported only linear on-states. ${ }^{[8,25,31]}$ However, reversal of the $\mathrm{Ta}_{2} \mathrm{O}_{5-x}$ and $\mathrm{TaO}_{y}$ deposition sequence means the graphene $\mathrm{BE}$ will be exposed to fundamentally different environments - an inert $\mathrm{Ar}$ environment if the $\mathrm{Ta}_{2} \mathrm{O}_{5-x}$ film is deposited first and an oxygen plasma if the $\mathrm{TaO}_{\mathrm{y}}$ film is deposited first. It is likely that in the latter case during reactive sputtering of the $\mathrm{TaO}_{\mathrm{y}}$ film the graphene $\mathrm{BE}$ is oxidized (functionalized) as it was heated $\left(400{ }^{\circ} \mathrm{C}\right)$ and subjected to an oxidative $\mathrm{Ar} / \mathrm{O}_{2}$ plasma. Consequently, the non-linear behavior in the MLG/ $\mathrm{Ta}_{2} \mathrm{O}_{5-x} / \mathrm{TaO}_{\mathrm{y}} / \mathrm{MLG}$ devices likely originates from the functionalized graphene $\mathrm{BE}$.
To test this hypothesis, we measured the properties of the graphene $\mathrm{BEs}$ of the devices between two different bottom pads B1 and B2 (see Figure 1a). Figures 2a,b show the currentvoltage $(I-V)$ characteristics of the graphene BE after the MLG/ $\mathrm{Ta}_{2} \mathrm{O}_{5-x} / \mathrm{TaO}_{\mathrm{y}} / \mathrm{MLG}$ device fabrication, revealing a pronounced volatile threshold switching behavior in both positive and negative directions. That is, the device is switched to a low resistance state (LRS) when a sufficiently large voltage above a threshold $\left(V_{\text {th }}\right)$ is applied at either bias polarity, as exemplified in Figure 2b. However the resistance change is volatile and the LRS can only be maintained when a voltage above the hold voltage $\left(V_{h}\right)$ is applied, and the device will return to the original high resistance state (HRS) when the voltage drops below $V_{\mathrm{h}}$. Similar threshold switching behavior can be observed on both sides of the bias polarity, consistent with the expectations from the symmetric test structure consisting of just the graphene BE. In addition, the resistance of the graphene BE prior to switching is also significantly increased compared to the pristine graphene case (e.g. $\sim 20 \mathrm{k} \Omega$ vs. $\sim 2 \mathrm{k} \Omega$ ). In contrast, the graphene electrodes without functionalization (e.g. T1-T2 or B1-B2 prior to oxide deposition) exhibits typical Ohmic conduction expected from pristine graphene electrodes, as shown in Figure 2c. Additionally, Ohmic conduction was observed in the BE test structures in $\mathrm{MLG} / \mathrm{TaO}_{\mathrm{y}} / \mathrm{Ta}_{2} \mathrm{O}_{5-x} / \mathrm{MLG}$ devices even after oxide deposition (Figure 2d), without any threshold switching behavior. These results are consistent with the hypothesis of spontaneous functionalization (oxidation) of graphene during reactive $\mathrm{TaO}_{y}$ deposition for the $\mathrm{MLG} / \mathrm{Ta}_{2} \mathrm{O}_{5-x} / \mathrm{TaO}_{\mathrm{y}} /$ MLG devices. The spontaneous functionalization has largely modified the physical/chemical property of the graphene BEs, 
(a)

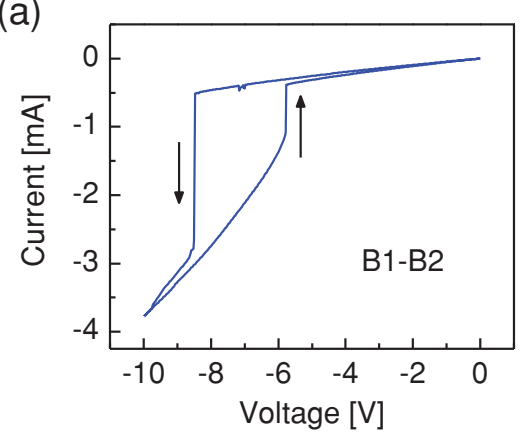

(d)

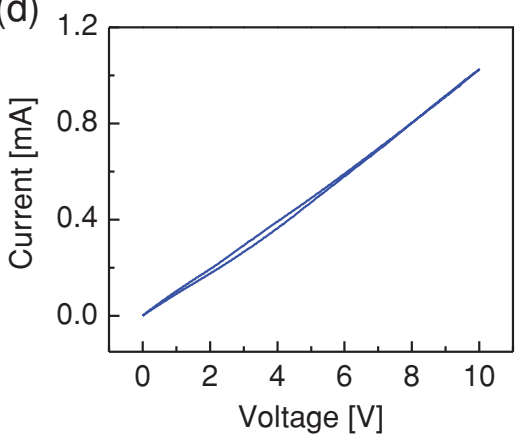

(b)

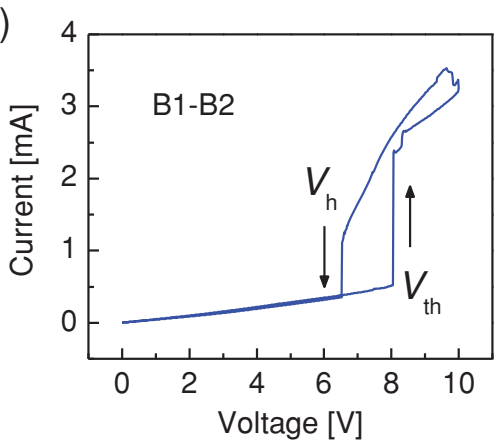

(e)

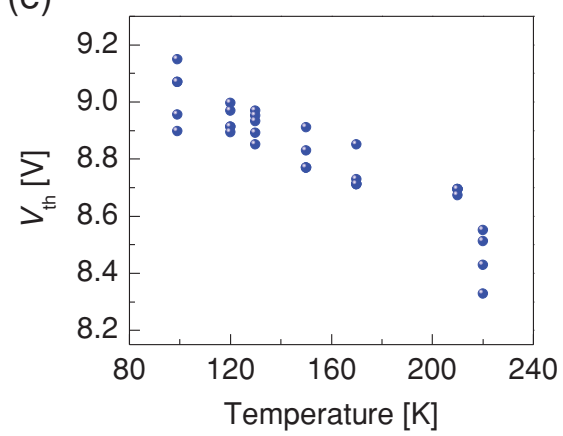

(c)

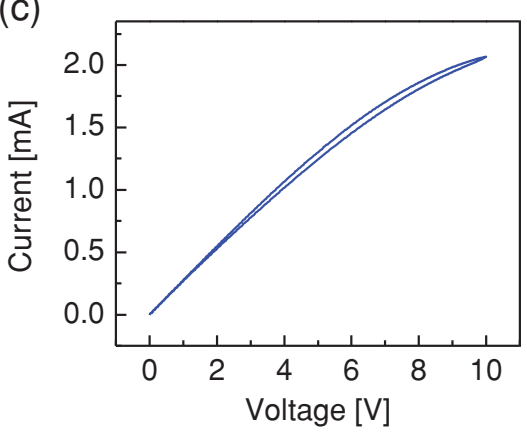

(f)

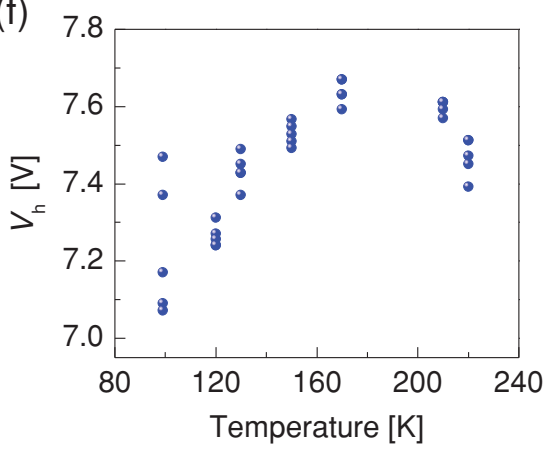

Figure 2. Threshold switching characteristics in functionalized graphene electrode. (a, b) Threshold switching behavior in negative (a) and positive (b) voltage regions measured from a graphene $\mathrm{BE}(\mathrm{B} 1-\mathrm{B} 2)$ in a $\mathrm{MLG} / \mathrm{Ta}_{2} \mathrm{O}_{5-x} / \mathrm{TaO}_{\mathrm{y}} / \mathrm{MLG}$ device. (c) $1-V$ characteristics of a graphene electrode without functionalization. (d) $I-V$ characteristics measured from a graphene BE (B1-B2) in a MLG/TaO $/ \mathrm{Ta}_{2} \mathrm{O}_{5-x} / \mathrm{MLG}$ device. (e, f) The threshold voltage and hold voltage $V_{\text {th }}(\mathrm{e})$ and $V_{\mathrm{h}}(\mathrm{f})$ for the functionalized graphene BE as a function of temperature.

enabling an unexpected threshold switching behavior in the graphene $\mathrm{BE}$.

Analogous to threshold switches ${ }^{[23,32]}$ or IMT switches ${ }^{[22]}$ that may be utilized as selector elements of RRAM, the graphene BEs with the pronounced threshold switching characteristics can effectively serve as a selector element in the MLG/ $\mathrm{Ta}_{2} \mathrm{O}_{5-x} /$ $\mathrm{TaO}_{\mathrm{y}} / \mathrm{MLG}$ system, therefore forming a 1 selector -1 resistor (1S1R) structure along with the memory component and displaying the nonlinear $I-V$ characteristics (Figure 1e). Notably since the selector element intrinsically resides in the atomically thin functionalized graphene BE and no additional films or device stacks are required, this $1 \mathrm{~S} 1 \mathrm{R}$ structure is highly compact, as schematically shown in Figure 1f. The ability to provide an intrinsic selector element, in combination with other unique properties offered by graphene, makes graphene an attractive material for RRAM devices.

Threshold switching components, e.g. the functionalized graphene $\mathrm{BE}$ in $\mathrm{MLG} / \mathrm{Ta}_{2} \mathrm{O}_{5-x} / \mathrm{TaO}_{y} / \mathrm{MLG}$ devices are promising candidates for selector elements, as the current is strongly suppressed at low bias conditions. Threshold switching is normally believed to be driven by electronic processes (e.g. electron trapping) which lead to volatile switching ${ }^{[33]}$ vs. nonvolatile memory switching which involves the redistribution of ions. ${ }^{[2,13]}$ Other threshold switching behaviors have been found to be caused by temperature changes due to Joule heating. ${ }^{[22,34]}$ For the case of functionalized graphene, theoretical calculations suggest that the interaction between oxygen and graphene mainly involves two effects: physisorption and chemisorption/ oxidation. ${ }^{[18]}$ Since physisorbed oxygen molecules were found to result in upshift of the Dirac point and p-doping which will lead to decreased resistance, ${ }^{[35,36]}$ the observed increase in graphene BE resistance and the associated non-Ohmic behavior suggest that they must be from chemical origins.

In the case of chemisorption/oxidation, a $s p^{3}$ chemical derivative of graphene is obtained with a high sheet resistance that can reach the $M \Omega-G \Omega$ regime if the graphene sheet is fully oxidized. ${ }^{[36]}$ In the present case, however, the resistance of the graphene BE was only increased by a factor of $\sim 10$ after device fabrication, to $\sim 20 \mathrm{k} \Omega$ (supplementary Figure S2). It hence suggests the graphene $\mathrm{BE}$ in $\mathrm{MLG} / \mathrm{Ta}_{2} \mathrm{O}_{5-x} / \mathrm{TaO}_{y} / \mathrm{MLG}$ devices was only partially oxidized, that is, a certain concentration of epoxide groups were grafted onto the basal plane of the graphene sheet, ${ }^{[18]}$ as schematically illustrated in Figure 3a. Indeed, recent theoretical and experimental studies on graphene films subjected to ozone treatments show similar transition to an insulator behavior when only a fraction of graphene is functionalized with epoxide groups. ${ }^{[18,36]}$

This hypothesis was verified by Raman spectroscopy on the functionalized graphene sample, as shown in Figure 3b. Generally speaking, in Raman spectra of carbon based materials, the main features are the $G$ peak corresponding to optical $E_{2 g}$ phonons, the D peak caused by defect-activated breathing-like modes, and the 2D peak as overtone of the $\mathrm{D}$ peak, with locations around 1600,1300 , and $2650 \mathrm{~cm}^{-1}$, respectively. ${ }^{[17,37,38]}$ The intensity of the $\mathrm{D}$ peak thus provides a convenient measure for the amount of disorder in graphene. ${ }^{[37,38]}$ Compared with pristine graphene, the largely increased D peak intensity in functionalized graphene (Figure $3 \mathrm{~b}$ ) is in agreement with the increased amount of disorder/defects caused by $s p^{3}$ bonding (epoxide groups). The decrease of the $2 \mathrm{D}$ peak 


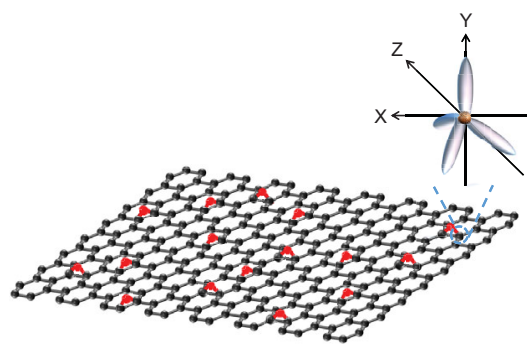

(a)

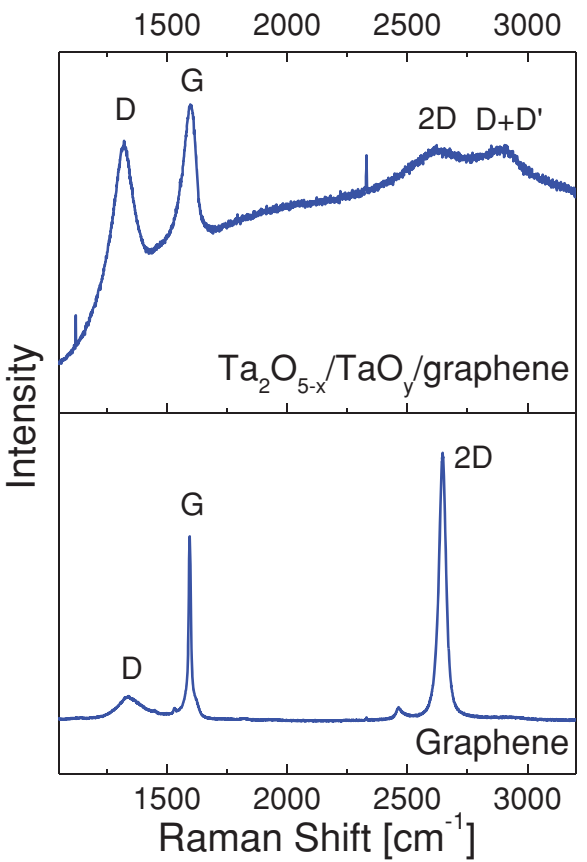

(b)

Figure 3. Functionalization of graphene via oxidation. (a) Schematic of a partially oxidized graphene with epoxide groups grafted on the basal plane of the graphene sheet. (b) Raman spectra of pristine graphene (bottom panel) and functionalized graphene (top panel).

intensity relative to the $\mathrm{G}$ peak and the onset of a combination mode D + D' around $2900 \mathrm{~cm}^{-1}$ are also consistent with those observed in hydrogenated graphene with similar $s p^{3}$ hybridizations. ${ }^{[17]}$

The conduction mechanism of the functionalized graphene $\mathrm{BE}$ in the HRS regime was further examined through temperature dependent studies (Figure 4). Figure 4a shows the read current of the HRS state of the functionalized graphene $\mathrm{BE}$ as a function of temperature $(I-T)$, revealing a monotonically increasing trend of current as temperature increases. Such behavior is consistent with a thermal activation transport process and contrasts with the metallic behavior of pristine graphene electrodes. By fitting the $I-T$ data in Figure 4a with different models, the best fitting in Figure $4 \mathrm{~d}$ reveals a linear relationship between $\ln I$ and $T^{-1 / 3}$, suggesting variable range hopping (VRH) as a plausible charge-transport mechanism in the partially oxidized graphene (also see supplementary Figure S3). ${ }^{[39]}$ In general, VRH is fulfilled by consecutive inelastic tunneling processes between localized states and is usually observed in disordered systems, including carbonbased materials with high levels of disorder such as amorphous carbon, ${ }^{[40]}$ partially reduced graphene oxide ${ }^{[41]}$ and hydrogenated graphene. ${ }^{[17]}$ The VRH conduction process is consistent with the increased disorder observed from Raman studies in that the partial oxidation has modified graphene from a highly ordered crystalline state to a disordered state with increased amount of defects. Based on these observations, we suggest charge transport in partially oxidized graphene BE consists of electron hopping between the intact graphene regions separated by the $s p^{3}$ hybridized regions. The temperature-dependent characteristics of VRH can be described as

$$
I=I_{0} \exp \left(-\frac{T_{0}}{T}\right)^{\frac{1}{d+1}}
$$

where $d$ is the dimensionality of the sample. ${ }^{[39]}$ The linear relationship between $\ln I$ and $T^{-1 / 3}$ in Figure $4 \mathrm{~d}$ is thus consistent with the 2D structure of graphene $(d=2)$.

In stark contrast to the behavior of the HRS, the temperature dependence of the LRS of the same functionalized graphene BE after threshold switching shows a metallic behavior (Figure 4b), i.e. the current decreases as the temperature is increased in the LRS. A read voltage above $V_{\text {th }}$ was adopted in this case to maintain the device in LRS during the measurement. Additionally, characterizations of graphene BEs before any oxide deposition also show metallic behavior expected from pristine graphene (Figure 4c) and confirm that the VRH in functionalized graphene is indeed a result of partial oxidation.

Based on these analyses, we hypothesize that the threshold switching behavior observed in functionalized graphene is driven by the nonequilibrium population of high-mobility shallow traps at high electric fields, with the defects introduced during graphene oxidation. The existence of such defects was verified by the Raman results (Figure 3b) and the appearance of VRH conduction in HRS (Figure 4a,d). Similar switching behavior has been analytically modeled based on trap-limited conduction $^{[33]}$ and experimentally observed in other disordered oxide ${ }^{[42-45]}$ or chalcogenide ${ }^{[33]}$ systems. Therefore, the threshold switching behavior in functionalized graphene can be explained as follows. The epoxide groups in the functionalized graphene electrode act as deep-level traps for conduction electrons, leading to a VRH with low conductance. At sufficiently high voltages, the high-energy electrons will be able to access higher-level shallow traps, resulting in a dramatic increase in conductivity and current, corresponding to the switching from the HRS to the LRS. ${ }^{[33]}$ Similar process takes place in either voltage polarity so the switching is unipolar. Figure 2e,f shows $V_{\text {th }}$ and $V_{\mathrm{h}}$ as a function of temperature, where relatively small changes can be observed across a temperature range of $\sim 120 \mathrm{~K}$. This weak T-dependence in threshold voltage is a signature of electronic process such as charge hopping between traps which is mainly field-driven, ${ }^{[46]}$ further supporting the above interpretation. The volatile nature of electron trapping/detrapping in the functionalized graphene $\mathrm{BE}$ and the nonvolatile nature of $\mathrm{V}_{\mathrm{O}} \mathrm{S}$-migration induced bipolar switching in the tantalum oxide layers lead to the formation of a 1S1R structure (Figure 1f), resulting in the overall $I-V$ characteristics of $\mathrm{MLG} / \mathrm{Ta}_{2} \mathrm{O}_{5-x} /$ $\mathrm{TaO}_{\mathrm{y}} / \mathrm{MLG}$ devices with highly nonlinear on state. 
(a)

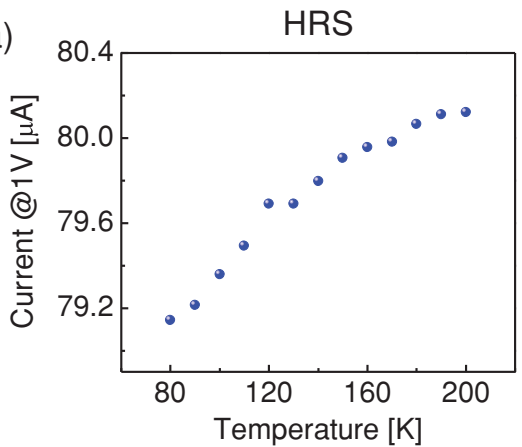

(d)

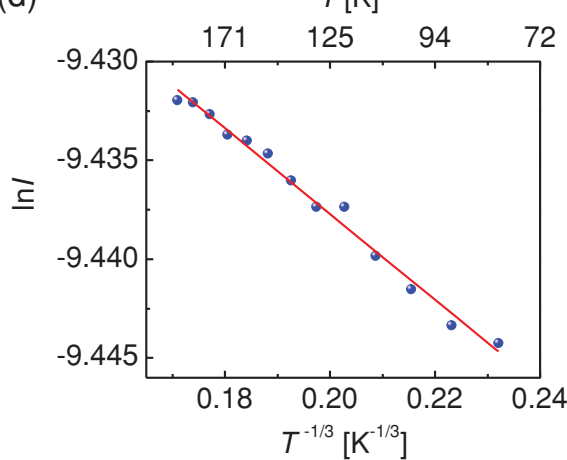

(b)

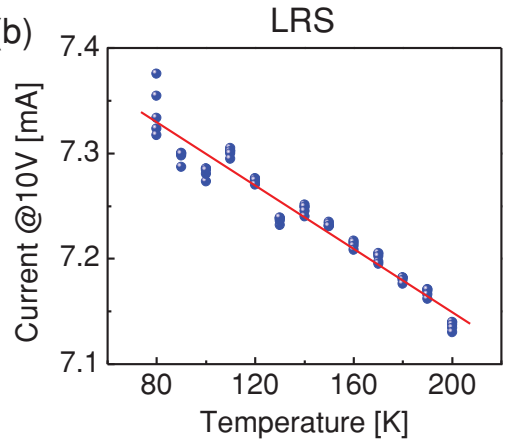

(e)

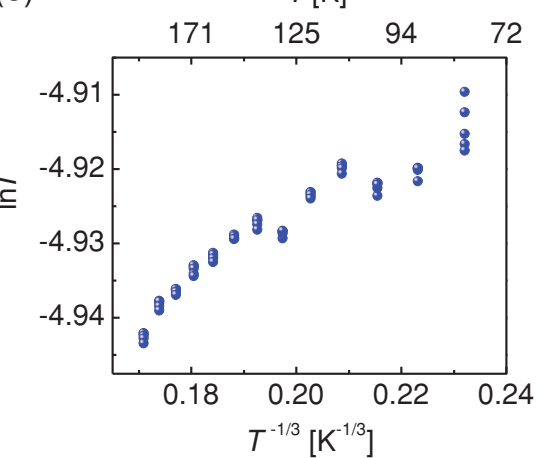

(c)

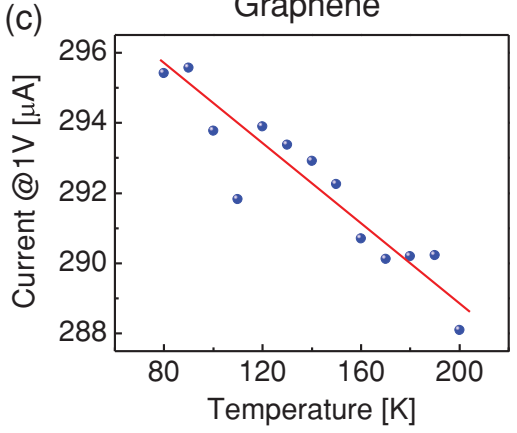

(f)

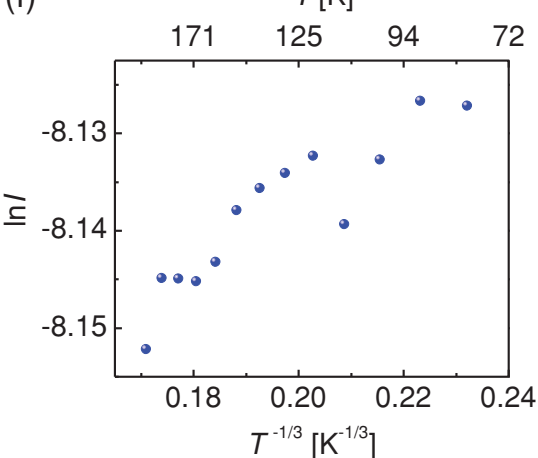

Figure 4. Temperature-dependent transport in graphene electrodes. (a) I-T characteristics obtained from the HRS state of the functionalized graphene BE. Read voltage: $1 \mathrm{~V}$. (b) I-T characteristics obtained from the LRS state of the same device. A higher read voltage of $10 \mathrm{~V}$ was used to keep the device in LRS. The solid line is linear fit. (c) I-T characteristics obtained from a pristine graphene electrode. Read voltage: $1 \mathrm{~V}$. The solid line is linear fit. (d-f) In I vs. $T^{-1 / 3}$ plots of the $I-T$ characteristics obtained from the HRS, LRS, and pristine graphene electrodes, respectively. The solid line in (d) is linear fit.

Additional control experiments were performed to show that partially oxidized graphene electrodes can indeed exhibit volatile threshold switching. The experiments were carried out by directly annealing the graphene electrodes in a controlled $\mathrm{O}_{2}$ environment at different temperatures. As shown in Figure 5a, when the annealing temperature was incrementally increased, the resistance of the graphene electrodes was first decreased $\left(300-500{ }^{\circ} \mathrm{C}\right)$, suggestive of dominant $p$-type doping effect due to physisorption at relatively low temperatures. ${ }^{[36]}$
When the annealing temperature was increased further, significant increase in resistance was observed instead $\left(600{ }^{\circ} \mathrm{C}\right)$, implying graphene oxidation has become the dominant effect. ${ }^{[36]}$ The higher temperature required to oxidize graphene during annealing is due to the less oxidative environment compared with the oxygen plasma during reactive sputtering. Notably, clear resistive switching was also observed in the control devices that were annealed at $600{ }^{\circ} \mathrm{C}$ in oxygen (Figure $5 \mathrm{a}$ ). Other factors such as metal pad oxidation as the
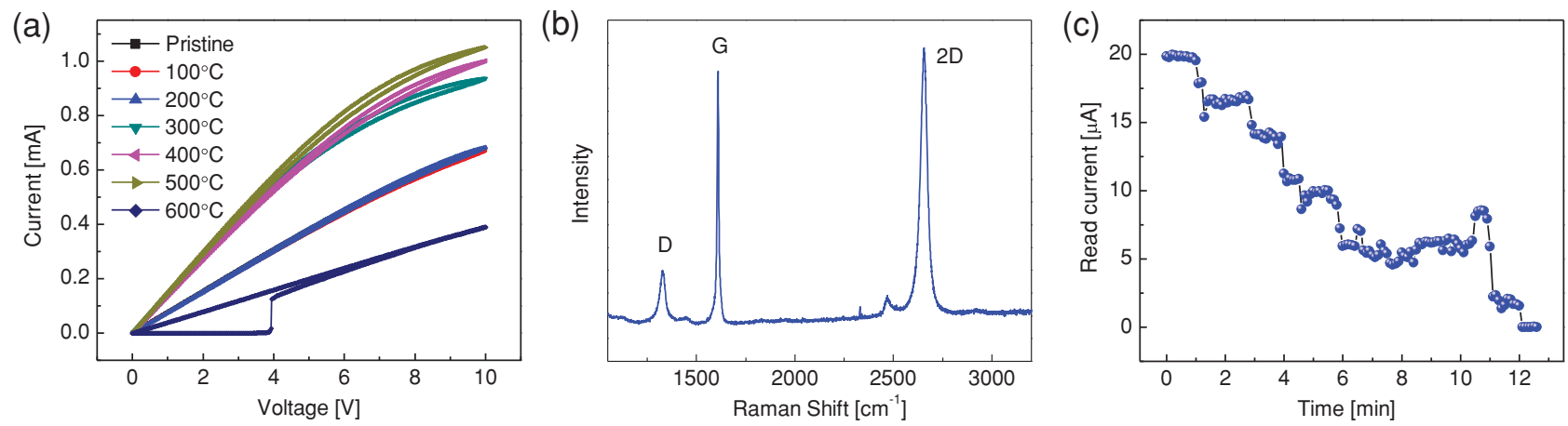

Figure 5. Resistive switching in oxygen annealed graphene. (a) $I-V$ characteristics of graphene electrodes as a function of annealing temperature. An increase in resistance and clear resistive switching behavior were observed for devices annealed at $600^{\circ} \mathrm{C}$, while a decrease in resistance was observed at low temperature annealing. (b) Raman spectrum of a graphene film after oxidation in $\mathrm{O}_{2}$. Similar to the functionalized graphene case, increased intensity of D peak was also observed. The still lower D peak intensity compared to that of the functionalized graphene in Figure $3 \mathrm{~b}$ is likely due to the absence of structural defects caused by ion bombardments in the functionalized graphene. (c) Retention behavior of the LRS of the oxygen annealed device. Read voltage: $1 \mathrm{~V}$. The short retention indicates a volatile nature. 
origin of resistive switching were safely excluded (supplementary Figure S4). Furthermore, the most pronounced change in Raman spectrum, i.e. the increased D peak intensity implying high degree of disorder, was also reproduced in the annealed graphene samples (Figure 5b). These results confirm that the switching behavior of the functionalized graphene electrodes in $\mathrm{MLG} / \mathrm{Ta}_{2} \mathrm{O}_{5-x} / \mathrm{TaO}_{y} / \mathrm{MLG}$ devices was indeed induced by the oxidation of graphene.

It should be noted that the functionalized graphene electrodes showed clearly volatile threshold switching behavior (e.g. Figure 2a,b), while the annealed graphene samples offered some level of retention (Figure $5 \mathrm{a}$ ). Figure $5 \mathrm{c}$ plots the retention behavior of the annealed graphene samples, where short retention times of $\sim$ minutes were typically observed with some devices showing much shorter retention time $\sim$ seconds. The stepwise variations in Figure $5 \mathrm{c}$ on top of the overall current decay could be explained by the stochastic change in the conduction path due to persistent trapping and detrapping of electrons leading to the rearrangement of the network composed of occupied traps. ${ }^{[47,48]}$ Such behaviors have been successfully modeled for percolation networks with competing defect generation and defect recovery mechanisms, ${ }^{[48,49]}$ again supporting electron trapping as the mechanism of the observed switching effect in oxidized graphene electrodes. The difference in retention time between the functionalized graphene electrodes in the $\mathrm{MLG} / \mathrm{Ta}_{2} \mathrm{O}_{5-x} / \mathrm{TaO}_{\mathrm{y}} / \mathrm{MLG}$ devices and the annealed graphene samples may be explained by the different interfaces in the two types of devices, as the $\mathrm{TaO}_{\mathrm{y}}$ layer passivating the graphene film could change the energy profile of the electron traps and make it easier for the trapped electrons to escape in the $\mathrm{MLG} / \mathrm{Ta}_{2} \mathrm{O}_{5-x} / \mathrm{TaO}_{\mathrm{y}} / \mathrm{MLG}$ devices.

In summary, we demonstrated oxide-based resistive memory devices with graphene electrodes that offer an internal selector element. The spontaneous functionalization of graphene during device fabrication results in volatile threshold switching in the functionalized graphene electrode and contributes to the overall highly nonlinear on-state characteristics of the resistive memory. The successful integration of the selector and the memory components with graphene electrodes offers an attractive option for stackable 3D RRAM integrations and flexible electronics. The findings of spontaneous oxidation of graphene and related resistive switching characteristics will also enrich understanding on electrical transport of graphene and graphene derivatives.

\section{Experimental Section}

Graphene transfer: Single layer graphene films was grown on copper foils by CVD. The films were then coated with PMMA resist (Microchem) and left in iron (III) nitrate (Sigma Aldrich) solution $(0.05 \mathrm{~g} / \mathrm{ml})$ for 12 hours to completely dissolve the copper foil. The PMMA/graphene stack was then transferred onto a glass substrate, and the PMMA coating was subsequently removed with acetone, followed by thorough rinsing with deionized (DI) water. The process was repeated several times to obtain multiple layers of graphene. The property of the pristine graphene and functionalized graphene sheets were characterized by Raman spectroscopy (633 nm, Renishaw inVia Raman Microscope).

Electrical measurements: The electrical measurements in this work were conducted using a customized measurement setup in combination with a temperature-variable probe station (Desert Cryogenics TTP4). The bilayer RRAM devices with graphene electrodes were measured in air ambient with the bottom electrode grounded, while the temperaturedependence characterizations and measurements on annealed graphene samples were performed in vacuum.

\section{Supporting Information}

Supporting Information is available from the Wiley Online Library or from the author.

\section{Acknowledgements}

This work was supported in part by the AFOSR through MURI grant FA9550-12-1-0038 and grant FA9550-12-1-0441, and by the National Science Foundation grant ECCS-0954621. Graphene synthesis was supported by the National Science Foundation Scalable Nanomanufacturing Program (DMR-1120187). This work used the Lurie Nanofabrication Facility at the University of Michigan, a member of the National Nanotechnology Infrastructure Network (NNIN) funded by NSF.

Received: January 17, 2014

Revised: February 6, 2014

Published online: March 18, 2014

[1] R. Waser, M. Aono, Nat. Mater. 2007, 6, 833

[2] D. B. Strukov, G. S. Snider, D. R. Stewart, R. S. Williams, Nature 2008, 453, 80 .

[3] L. O. Chua, IEEE Trans. Circuit Theory 1971, 18, 507.

[4] J. J. Yang, D. B. Strukov, D. R. Stewart, Nat. Nanotechnol. 2013, 8, 13.

[5] J. Borghetti, G. S. Snider, P. J. Kuekes, J. J. Yang, D. R. Stewart, R. S. Williams, Nature 2010, 464, 873.

[6] Y. Yang, P. Gao, S. Gaba, T. Chang, X. Pan, W. Lu, Nat. Commun. 2012, 3, 732

[7] T. Ohno, T. Hasegawa, T. Tsuruoka, K. Terabe, J. K. Gimzewski, M. Aono, Nat. Mater. 2011, 10, 591.

[8] M.-J. Lee, C. B. Lee, D. Lee, S. R. Lee, M. Chang, J. H. Hur, Y.-B. Kim, C.-J. Kim, D. H. Seo, S. Seo, U. I. Chung, I.-K. Yoo, K. Kim, Nat. Mater. 2011, 10, 625.

[9] A. C. Torrezan, J. P. Strachan, G. Medeiros-Ribeiro, R. S. Williams, Nanotechnology 2011, 22, 485203.

[10] B. Govoreanu, G. S. Kar, Y. Y. Chen, V. Paraschiv, S. Kubicek, A. Fantini, I. P. Radu, L. Goux, S. Clima, R. Degraeve, N. Jossart, O. Richard, T. Vandeweyer, K. Seo, P. Hendrickx, G. Pourtois, H. Bender, L. Altimime, D. J. Wouters, J. A. Kittl, M. Jurczak, IEEE Technical Digest International Electron Devices Meeting 2011, 31, 6.

[11] J. W. Seo, J. W. Park, K. S. Lim, J. H. Yang, S. J. Kang, Appl. Phys. Lett. 2008, 93, 223505.

[12] S. Kim, H. Y. Jeong, S. K. Kim, S.-Y. Choi, K. J. Lee, Nano Lett. 2011, $11,5438$.

[13] R. Waser, R. Dittmann, G. Staikov, K. Szot, Adv. Mater. 2009, 21, 2632.

[14] J. Yao, J. Lin, Y. Dai, G. Ruan, Z. Yan, L. Li, L. Zhong, D. Natelson, J. M. Tour, Nat. Commun. 2012, 3, 1101.

[15] A. K. Geim, K. S. Novoselov, Nat. Mater. 2007, 6, 183.

[16] T. S. Sreeprasad, V. Berry, Small 2013, 9, 341.

[17] D. C. Elias, R. R. Nair, T. M. G. Mohiuddin, S. V. Morozov, P. Blake, M. P. Halsall, A. C. Ferrari, D. W. Boukhvalov, M. I. Katsnelson, A. K. Geim, K. S. Novoselov, Science 2009, 323, 610. 
[18] N. Leconte, J. Moser, P. Ordejon, H. Tao, A. Lherbier, A. Bachtold, F. Alsina, C. M. Sotomayor Torres, J.-C. Charlier, S. Roche, ACS Nano 2010, 4, 4033.

[19] C. L. He, F. Zhuge, X. F. Zhou, M. Li, G. C. Zhou, Y. W. Liu, J. Z. Wang, B. Chen, W. J. Su, Z. P. Liu, Y. H. Wu, P. Cui, R.-W. Li, Appl. Phys. Lett. 2009, 95, 232101.

[20] H. Y. Jeong, J. Y. Kim, J. W. Kim, J. O. Hwang, J.-E. Kim, J. Y. Lee, T. H. Yoon, B. J. Cho, S. O. Kim, R. S. Ruoff, S.-Y. Choi, Nano Lett. 2010, 10, 4381

[21] Y. Chen, B. Zhang, G. Liu, X. Zhuang, E. -T. Kang, Chem. Soc. Rev. 2012, 41, 4688 .

[22] M.-J. Lee, Y. Park, D. S. Suh, E. H. Lee, S. Seo, D. C. Kim, R. Jung, B. S. Kang, S. E. Ahn, C. B. Lee, D. H. Seo, Y. K. Cha, I. K. Yoo, J. S. Kim, B. H. Park, Adv. Mater. 2007, 19, 3919.

[23] M.-J. Lee, D. Lee, S.-H. Cho, J.-H. Hur, S.-M. Lee, D. H. Seo, D.-S. Kim, M.-S. Yang, S. Lee, E. Hwang, M. R. Uddin, H. Kim, U. I. Chung, Y. Park, I.-K. Yoo, Nat. Commun. 2013, 4, 2629.

[24] S. Lee, K. Lee, C.-H. Liu, Z. Zhong, Nanoscale. 2012, 4, 639.

[25] Y. Yang, S. Choi, W. Lu, Nano Lett. 2013, 13, 2908.

[26] Y. Yang, P. Sheridan, W. Lu, Appl. Phys. Lett. 2012, 100, 203112.

[27] G.-S. Park, Y. B. Kim, S. Y. Park, X. S. Li, S. Heo, M.-J. Lee, M. Chang, J. H. Kwon, M. Kim, U. I. Chung, R. Dittmann, R. Waser, K. Kim, Nat. Commun. 2013, 4, 2382.

[28] J. J. Yang, M. X. Zhang, M. D. Pickett, F. Miao, J. P. Strachan, W.-D. Li, W. Yi, D. A. A. Ohlberg, B. J. Choi, W. Wu, J. H. Nickel, G. Medeiros-Ribeiro, R. S. Williams, Appl. Phys. Lett. 2012, 100, 113501.

[29] W. Lee, J. Park, S. Kim, J. Woo, J. Shin, G. Choi, S. Park, D. Lee, E. Cha, B. H. Lee, H. Hwang, ACS Nano 2012, 6, 8166.

[30] J.-J. Huang, Y.-M. Tseng, C.-W. Hsu, T.-H. Hou, IEEE Electron Device Lett. 2011, 32, 1427.

[31] J. J. Yang, M. X. Zhang, J. P. Strachan, F. Miao , M. Pickett, R. D. Kelley, G. Medeiros-Ribeiro, R. S. Williams, Appl. Phys. Lett. 2010, 97, 232102.

[32] D. Kau, S. Tang, I. V. Karpov, R. Dodge, B. Klehn, J. A. Kalb J. Strand, A. Diaz, N. Leung, J. Wu, S. Lee, T. Langtry, K.-W. Chang, C. Papagianni, J. Lee, J. Hirst, S. Erra, E. Flores, N. Righos,
H. Castro, G. Spadini, IEEE Technical Digest International Electron Devices Meeting 2009, 617.

[33] D. lelmini, Y. Zhang, J. Appl. Phys. 2007, 102, 054517.

[34] M. D. Pickett, G. Medeiros-Ribeiro, R. S. Williams, Nat. Mater. 2013, 12, 114

[35] S. Jandhyala, G. Mordi, B. Lee, G. Lee, C. Floresca, P.-R. Cha, J. Ahn, R. M. Wallace, Y. J. Chabal, M. J. Kim, L. Colombo, K. Cho, J. Kim, ACS Nano 2012, 6, 2722.

[36] J. Yuan, L.-P. Ma, S. Pei, J. Du, Y. Su, W. Ren, H.-M. Cheng, ACS Nano 2013, 7, 4233.

[37] A. C. Ferrari, J. Robertson, Phys. Rev. B 2000, 61, 14095.

[38] F. Tuinstra, J. L. Koenig, J. Chem. Phys. 1970, 53, 1126.

[39] N. Mott, E. Davis, in Electronic Processes in Non-crystalline Materials, Clarendon press, Oxford, 1979.

[40] J. Robertson, Adv. Phys. 1986, 35, 317.

[41] C. Gómez-Navarro, R. T. Weitz, A. M. Bittner, M. Scolari, A. Mews, M. Burghard, K. Kern, Nano Lett. 2007, 7, 3499.

[42] A. Odagawa, H. Sato, I. H. Inoue, H. Akoh, M. Kawasaki, Y. Tokura, T. Kanno, H. Adachi, Phys. Rev. B 2004, 70, 224403.

[43] A. Chen, S. Haddad, Y. C. Wu, T. N. Fang, Z. Lan, S. Avanzino, S. Pangrle, M. Buynoski, M. Rathor, W. D. Cai, N. Tripsas, C. Bill, M. VanBuskirk, M. Taguchi, IEEE Technical Digest International Electron Devices Meeting 2005, 765.

[44] D. S. Shang, Q. Wang, L. D. Chen, R. Dong, X. M. Li, W. Q. Zhang, Phys. Rev. B 2006, 73, 245427.

[45] Q. Liu, W. H. Guan, S. B. Long, R. Jia, M. Liu, J. N. Chen, Appl. Phys. Lett. 2008, 92, 012117.

[46] A. B. K. Chen, S. G. Kim, Y. Wang, W.-S. Tung, I. W. Chen, Nat. Nanotechnol. 2011, 6, 237.

[47] R. Soni, P. Meuffels, G. Staikov, R. Weng, C. Kuegeler, A. Petraru M. Hambe, R. Waser, H. Kohlstedt, J. Appl. Phys. 2011, 110, 054509.

[48] N. Das, Y. Y. Xue, Y. Q. Wang, C. W. Chu, IEEE Non-Volatile Memory Technology Symposium 2009, 28.

[49] C. Pennetta, G. Trefán, L. Reggiani, AIP Conf. Proc. 2000, 511, 447. 